\title{
REGISTROS MÁS OCCIDENTALES DE CYRTOPHORA CITRICOLA (FORSKÅL, 1775) (ARANEAE: ARANEIDAE) EN CUBA
}

\author{
Yulianis Martín-Castejón ${ }^{1}$ y Alexander Sánchez-Ruiz ${ }^{2}$
}

\author{
'Centro de Investigaciones de Medio Ambiente de Camagüey, Cuba. \\ yulianis@cimac.cu \\ ${ }^{2}$ Centro Oriental de Ecosistemas y Biodiversidad, Santiago de Cuba, Cuba. \\ alex@bioeco.ciges.inf.cu
}

\section{RESUMEN}

Se amplía la distribución conocida de Cyrtophora citricola (Forskål, 1775) a la provincia de Camagüey en Cuba. Estos constituyen, hasta el momento, los registros más occidentales de esta especie exótica invasora en Cuba.

Palabras clave: Cyrtophora, exótica, invasora, Cuba.

\section{ABSTRACT}

The known distribution of Cyrtophora citricola (Forskål, 1775) is extended to Camagüey Province in Cuba. At the moment, these are the most western records of this invasive exotic species in Cuba.

Keywords: Cyrtophora, exotic, invasive, Cuba.

La araña Cyrtophora citricola (Forskål, 1775), comúnmente llamada araña africana, es una especie invasora que ha ido ampliando sus poblaciones en varias zonas de América. Hasta el momento se conoce su presencia en Colombia (Levi, 1997), Sur de la Florida (Mannion et al., 2002), Minas Gerais en Brasil (Soares-Álvares \& De María, 2004), Cuba (Alayón-García, 2003; Sánchez-Ruizy Teruel, 2006) y La Hispaniola (Alayón-García et al., 2001; Starr, 2005).

Esta especie fue registrada por primera vez para Cuba en los márgenes del río Toa, municipio Baracoa, Provincia Guantánamo (Alayón-García, 2003). Aparentemente, su introducción en el archipiélago cubano fue de forma natural, a partir de poblaciones procedentes de Haití que se dispersaron a través del Paso de los Vientos; utilizando un mecanismo de dispersión aérea denominado ballooning (aerostato), que utilizan generalmente esta y otras familias de arañas tejedoras. Posteriormente, la especie fue expandiendo sus poblaciones a casi toda la Región Oriental de Cuba (Sánchez-Ruiz y Teruel, 2006) utilizando definitivamente este mecanismo.

En julio del 2008, en un viaje de campo realizado a la Reserva Ecológica LimonesTuabaquey, en la Sierra de Cubitas, como parte de acciones de monitoreo ejecutadas por el Centro de Investigaciones de Medio Ambiente de Camagüey, fue colectado un ejemplar hembra de Cyrtophora citricola en la localidad del Bosque Domínguez (21 $\left.36^{\prime} 25^{\prime \prime} \mathrm{N}-77^{\circ} 45^{\prime} 36^{\prime \prime} \mathrm{W}\right)$. El ejemplar encontrado se hallaba en una densa tela que cubría una gran parte de las ramas de un individuo de Ocotea coriacea (Sw.) Britt. (Lauraceae) que aún no había alcanzado su tamaño definitivo. Posteriormente, en octubre del 2008, durante un Inventario Biológico Rápido desarrollado por especialistas del Centro Oriental de Ecosistemas y Biodiversidad y La Empresa Nacional para la Protección de la Flora y la Fauna en la Reserva Florística La Silla de Romano, Cayo Romano, se colectó otro ejemplar hembra de esta araña en una vegetación xerofítica $2 \mathrm{~km}$. al Oeste de Playa del Muerto (21 $\left.48^{\prime} 19^{\prime \prime} \mathrm{N}-77^{\circ} 27^{\prime} 01^{\prime \prime} \mathrm{W}\right)$.

Estos constituyen, hasta el momento, los registros más occidentales de esta especie invasora en Cuba. La presencia de esta araña en Sierra de Cubitas y en uno de los cayos de la costa norte del archipiélago (Fig. 1), demuestra que continúa invadiendo los ecosistemas cubanos con rumbo al occidente del país. Las telas de esta especie son muy fáciles de reconocer; no sólo por su gran extensión y aspecto sucio (debido a la gran cantidad de hojas y pequeñas ramas secas, ootecas y presas), sino por su particularidad de cubrir parcial o totalmente el sustrato seleccionado por la araña para construirlas. Se cree que las extensas y densas telas que construye pueden dañar la salud 


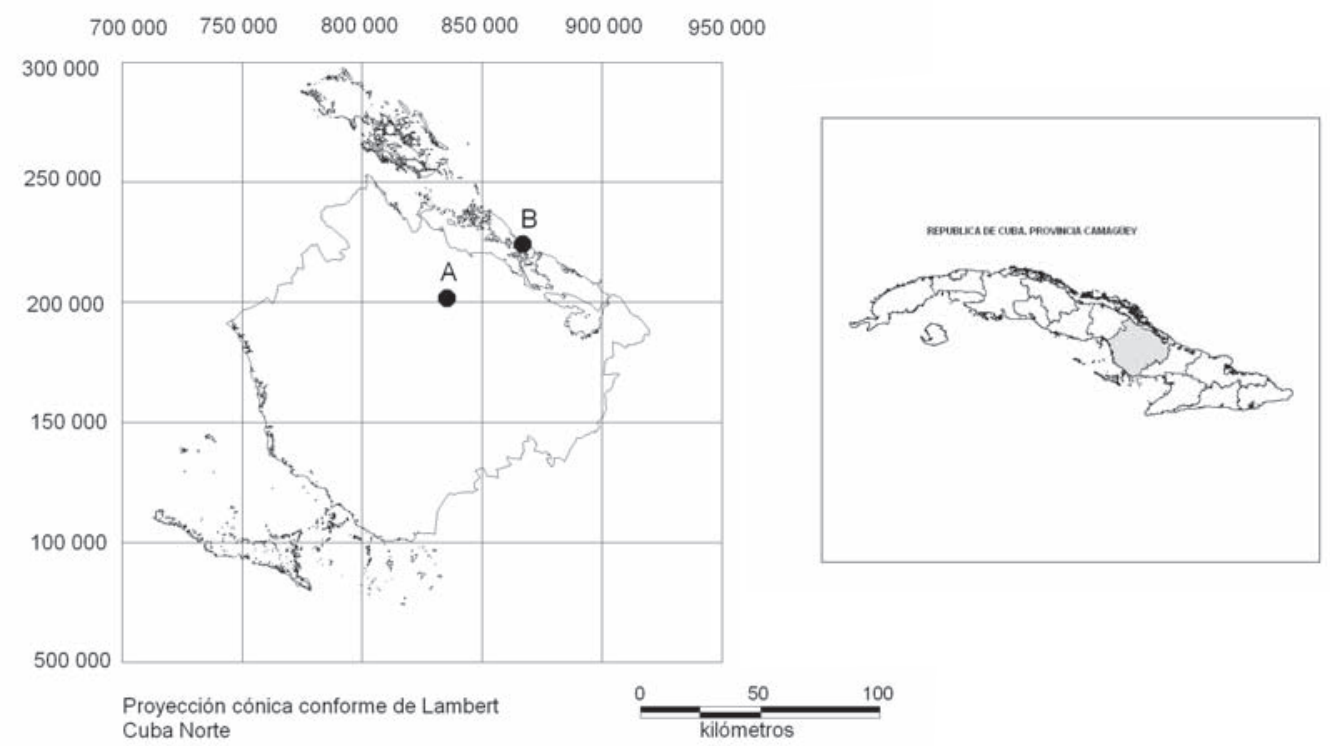

Figura 1. Localidades más occidentales de la distribución en Cuba de Cyrtophora citricola (Forskål, 1775). A. Reserva Ecológica Limones-Tuabaquey, Sierra de Cubitas; B. Reserva Florística La Silla de Romano, Cayo Romano.

de los árboles o arbustos donde se instala al bloquear el paso de la luz hacia las hojas de la planta (Soares-Álvares \& De María, 2004). En Cuba, durante la primera fase de expansión en la Región Oriental, afectó los cultivos de naranjas de las provincias de Santiago de Cuba y Guantánamo (Dr. Francisco Simon. Instituto de Sanidad Vegetal de Santiago de Cuba, com. pers.). La facilidad de conquistar y adaptarse a nuevos hábitats, la alta vagilidad y los daños que causa a la vegetación arbustiva, hacen de esta especie una de las arañas exóticas invasoras más peligrosas.

\section{AGRADECIMIENTOS}

A Giraldo Alayón (Museo Nacional de Historia Natural, La Habana, Cuba) quien facilitó datos sobre la distribución de la especie; a Eddy Martínez (Centro de Investigaciones de Medio Ambiente de Camagüey) por su ayuda en la elaboración de los mapas y a todos los trabajadores de la Reserva Ecológica Limones-Tuabaquey, en especial a Yunier Cardozo y Yeldis Abreu.

\section{LITERATURA CITADA}

Alayón-García, G. 2003. Cyrtophora citricola (Araneae: Araneidae), registro nuevo de araña para Cuba. Cocuyo 13: 14.

Alayón-García, G., L. de Armas y A. J. Abud. 2001. Presencia de Cyrtophora citricola (Forskål, 1775) (Araneae: Araneidae) en Las Antillas. Revista Ibérica de Aracnología. 4:9-10.

Levi, H. W. 1997. The American orb weavers genera Mecynogea, Manogea, Kapogea and Cyrtophora (Araneae: Araneidae). Bulletin Museum of Comparative Zoology, 155 (5): 215255.

Mannion, C., D. Amalin, J. Peña y G. B. Edwards. 2002. A new spider in Miami-Dade County: Cyrtophora citricola. Hort Newsletter, Univ. Florida. Extensión 2(2):3.

Sánchez-Ruiz, A. y R. Teruel. 2006. Acerca de la presencia de Cyrtophora citricola (Forskål, 1775) (Araneae: Araneidae) en Cuba. Boletín Sociedad Entomológica Aragonesa 38: 335-336.

Soares-Álvares, E. S. y M. de Maria. 2004. First record of Cyrtophora citricola (Forskål) in Brazil (Araneae: Araneidae) Rev. Brasileira Zool., 21: 155-156.

Starr, CH. K. 2005. Observaciones sobre Cyrtophora citricola (Araneae: Araneidae) en Haití. Cocuyo 15:15. 